\title{
Miocardite lúpica, da insuficiência cardíaca severa à normalização da função cardíaca - relato de caso
}

\section{Lupus myocarditis, from severe heart failure to normalization of function - case report}

\author{
Myrian Lopes Arruda Carneiro ${ }^{1}$. Tacilla Hanny de Souza Andrade ${ }^{2}$. Maurício Yukio Ogawa ${ }^{3}$. Fernanda \\ Nogueira Holanda Ferreira Braga ${ }^{4}$. \\ 1 Médica residente de clínica médica do Hospital Universitário Walter Cantídio (HUWC), Fortaleza, Ceará, Brasil. 2 Aluna de \\ medicina da Universidade Estadual do Ceará (UECE), Fortaleza, Ceará, Brasil. 3 Aluno de medicina da Universidade Federal \\ do Ceará (UFC), Fortaleza, Ceará, Brasil. 4 Mestrado em Patologia pela Universidade Federal do Ceará (UFC), médica chefe da \\ unidade de clínica médica do Hospital Universitário Walter Cantídio (HUWC), Fortaleza, Ceará, Brasil.
}

\section{RESUMO}

Lúpus Eritematoso Sistêmico (LES) é uma doença inflamatória sistêmica autoimune, acometendo o miocárdio em $10 \%$ dos pacientes, entretanto, costuma causar apenas disfunção leve. Apenas 5\% dos casos evoluem para insuficiência cardíaca (IC). Paciente do sexo feminino, 56 anos, apresentou, em janeiro de 2002, quadro de artrite em interfalangeanas de ambas as mãos, febre diária com calafrios e artralgias em ombros e joelhos, sendo diagnosticada com lúpus (fator antinuclear padrão salpicado - 1:160 e padrão periférico 1:80). Em novembro de 2003, paciente apresentou quadro de dispneia súbita aos pequenos esforços associada a dor retroesternal contínua e presença de bulhas hipofonéticas, sendo realizado ecodopplercardiograma, o qual evidenciou fração de ejeção $24 \%$. Foi diagnosticada com IC por miocardite lúpica com posterior recuperação da fração de ejeção após terapia de imunossupressão e otimimização da IC. Manteve quadro estável e acompanhamento. Em janeiro de 2016 apresentou novo quadro de IC por miocardite lúpica com nova recuperação da função cardíaca. Apesar da baixa incidência da IC por miocardite lúpica, o médico deve sempre manter o grau de alta suspeição quando diante de certo quadro clínico, por conta da elevada morbimortalidade.

Palavras-chave: Miocardite. Lúpus eritematoso sistêmico. Insuficiência cardíaca.

\section{ABSTRACT}

Systemic lupus erythematosus (SLE) is an autoimmune systemic inflammatory disease, affecting the myocardium in $10 \%$ of patients, although it usually causes only mild dysfunction. Only $5 \%$ of cases progress to heart failure (HF). A female patient, 56 years old, presented in January 2002, arthritis in interphalangeal in both hands, daily chills fever, and presented with arthralgia in the shoulders and knees, being diagnosed with lupus (antinuclear factor splattered - 1: 160 and peripheral standard 1:80). In November 2003, the patient presented sudden dyspnea on the small exertions associated with continuous retrosternal pain and presence of hypofontic sounds, with a Doppler echocardiogram (ECO), which showed a fraction of ejection fraction (EF) $=24 \%$. It was diagnosed with HF due to lupus myocarditis with subsequent recovery of the ejection fraction. The patient maintained stable frame and accompaniment. In January 2016 presented a new HF cardiac myocarditis with new recovery of cardiac function. Despite the low incidence of Chronic Heart Failure (CHF) due to lupus myocarditis, the physician should always maintain the degree of high suspicion when faced with a certain clinical picture, due to the high morbidity and mortality.

Keywords: Myocarditis. Lupus erythematosus systemic. Heart failure.

Autor correspondente: Myrian Lopes Arruda Carneiro, Av Desembargador Moreira 50, apartamento 1000, Meireles, Fortaleza, Ceará. CEP: 60170-000.Telefone: +55 85 98867-9861. E-mail: myrianarruda@hotmail.com

Conflito de interesses: Não há qualquer conflito de interesses por parte de qualquer um dos autores.

Recebido em: 03 Jan 2017; Revisado em: 01 Fev 2017; Aceito em: 25 Fev 2017. 


\section{INTRODUÇÃO}

OLúpusEritematosoSistêmico(LES)éumadoençainflamatória crônica autoimune cuja etiopatogenia envolve múltiplos genes, fatores hormonais e ambientais, predominando no sexo feminino. É uma doença pleomórfica com ampla variabilidade fenotípica de apresentação, gravidade e curso clínico e evolui habitualmente com períodos de atividade e remissão, podendo acometer qualquer órgão. ${ }^{1} \mathrm{O}$ coração pode ser acometido em todas suas camadas em forma de endocardite, pericardite e miocardite. A pericardite é a lesão ecocardiográfica mais comum no lúpus eritematoso sistêmico, sendo a manifestação cardíaca mais frequente ocorrendo em mais da metade dos pacientes. ${ }^{4}$ A miocardite é uma manifestação atípica, acometendo apenas $10 \%$ dos portadores do lúpus, ${ }^{2}$ cursando clinicamente de forma variada, podendo estar presente desde uma dor torácica até um quadro de insuficiência cardíaca por perda da função de ejeção. ${ }^{3}$ Apresentaremos um caso de uma paciente de 56 anos do sexo feminino que apresentou dois episódios de miocardite com recuperação da fração de ejeção em ambos os casos após o tratamento imunossupressor e otimização da insuficiência cardíaca (IC).

\section{RELATO}

Paciente do sexo feminino, 56 anos, do lar, casada, apresentou, em janeiro de 2002, quadro de artrite em interfalangeanas proximais e interfalangeanas distais de ambas mãos, febre diária com calafrios, artralgias em grandes articulações, como ombros, joelhos e cotovelos, buscando atendimento médico em maio de 2002. Além do quadro citado, apresentou dor torácica ventilatório dependente, mialgia, fator reumatoide não reagente e fator antinuclear reagente padrão salpicado 1:160 e padrão periférico 1:80. Foi diagnosticada com lúpus eritematoso sistêmico (LES) em junho de 2002 e iniciado tratamento com prednisona $20 \mathrm{mg} / \mathrm{dia}$, sulfato ferroso e ranitidina $150 \mathrm{mg} /$ dia e acompanhamento ambulatorial. Em maio de 2003, foi internada por apresentar quadro de adinamia, fraqueza muscular importante, vasculite em dedos das mãos (com áreas de necrose em segundo quirodáctilo), perda de peso, úlceras orais e odinofagia. Durante essa internação foi diagnostica uma pancitopenia (hemoglobina $8,4 \mathrm{~g} / \mathrm{dL}$, leucócitos $1.840 / \mathrm{mm}^{3}$ e plaquetas $109.000 / \mathrm{mm}^{3}$ ). Recebeu alta com prednisona $40 \mathrm{mg} /$ dia e ficou sendo acompanhada ambulatorialmente. Em agosto de 2003 iniciou o uso de azatioprina $50 \mathrm{mg} /$ dia como imunossupressor. Em novembro de 2003, paciente apresentou quadro de dispneia súbita aos pequenos esforços associada à dor retroesternal contínua e presença de bulhas hipofonéticas ao exame físico. Foi internada e realizado ecodopplercardiograma (ECO), o qual evidenciou fração de ejeção $(\mathrm{FE})=24 \%$ com hipocinesia difusa com aumento dos diâmetros intracavitários e déficit contrátil de grau importante. Foi feito o diagnóstico de insuficiência cardíaca (IC) por miocardite lúpica, sendo tratada com prednisona $1 \mathrm{mg} / \mathrm{kg} /$ dia e com terapia medicamentosa para insuficiência cardíaca. Em dezembro de 2004, o ECO demonstrou melhora do quadro cardíaco com FE subindo para 49\%. Novo ECO em fevereiro de 2006 mostrou função sistólica e diastólica do ventrículo esquerdo normais com FE de $76 \%$. Manteve quadro cardíaco estável e acompanhamento. Em setembro de 2009, devido apresentar quadro de nefrite lúpica leve (proteinúria de $24 \mathrm{~h}$ de $949 \mathrm{mg} / 24 \mathrm{~h}$; creatinina 1,1 $\mathrm{mg} / \mathrm{dL}$; consumo de complemento com C3 $=98 \mathrm{mg} / \mathrm{dL}$ e C4= $18 \mathrm{mg} / \mathrm{dL}$ ) foi introduzido micofenolato de mofetil $1 \mathrm{~g} /$ dia e suspendida a azatioprina. A paciente permaneceu em uso do micofenolato de mofetil $1 \mathrm{~g} /$ dia como imunossupressor até remissão da nefrite lúpica. No final do ano de 2015, voltou a usar a azatioprina como imunossupressor. Em janeiro de 2016, apresentou queixas de dispneia aos pequenos esforços e ortopnéia. Paciente havia suspendido a azatioprina há aproximadamente 2 a 3 meses devido a falta da medicação e vinha sem usar nenhuma medicação imunossupressora. Novo ECO evidenciou fração de ejeção de $28 \%$ e hipocinesia difusa importante com dilatação das câmaras esquerdas. Realizou cateterismo cardíaco que evidenciou lesão obstrutiva de $70 \%$ no terço médio da artéria descendente anterior; demais artérias normais; hipocontratilidade miocárdica difusa moderada. Realizou também ressonância miocárdica que evidenciou hipocinesia global com fração de ejeção reduzida e leve aumento da massa ventricular esquerda. Com o diagnóstico de miocardite foi instituído tratamento com pulsoterapia de metilprednisolona na dose de $1000 \mathrm{mg} /$ dia por 3 dias, seguido de prednisona $60 \mathrm{mg} /$ dia e terapia para IC (carvedilol, captopril, espironolactona, digoxina, furosemida em doses otimizadas). Paciente apresentou melhora do quadro clínico e novamente recuperação da função cardíaca com ECO de fevereiro de 2016 mostrando FE de 53\%. Atualmente, a paciente se encontra assintomática do ponto de vista da IC.

\section{DISCUSSÃO}

O envolvimento cardíaco do LES é relativamente comum (até $50 \%$ dos casos), podendo didaticamente ser dividido em doença vascular, pericárdica e em miocardite, a qual é isoladamente a mais rara. ${ }^{3}$ No que concerne à mesma, costuma normalmente se apresentar assintomaticamente e quando se apresenta de forma sintomática (cerca de 5-10\% dos casos de miocardite) manifesta-se desde forma mais leves, como febre até insuficiência cardíaca franca. ${ }^{3,4,5}$ Outras manifestações cardíacas da miocardite lúpica são dispneia, palpitações, dor torácica não relacionada ao esforço físico e distensão jugular. ${ }^{5}$ Um fato atípico do caso relatado é o fato de a paciente ter apresentado dois quadros de miocardite com insuficiência cardíaca com recuperação da função cardíaca.

Na literatura já existe uma grande correlação entre dispneia, dispneia paroxística noturna e ortopneia e a classificação funcional da New York Association (NYHA) com a miocardite lúpica. ${ }^{7}$ A paciente apresentou em ambos os casos dispneia significativa e classificação funcional NYHA 3, reforçando ainda mais o diagnóstico de miocardite lúpica.

Quanto ao diagnóstico, deve ser baseado em sinais clínicos de disfunção ventricular esquerda aliado a exclusão de outras causas, sendo o ECO um importante exame diagnóstico, pois corrobora ao não detectar alterações valvares/endocárdicas/ pericárdicas. ${ }^{4} \mathrm{O}$ padrão ouro para o diagnóstico da miocardite 
lúpica permanece sendo a biópsia endomiocárdica, porém é um procedimento invasivo e seu rendimento diagnóstico é baixo, em torno de 10 a 20\%. Dessa forma, o diagnóstico da miocardite no LES depende amplamente da suspeita clínica e dos achados ecocardiográficos, dentre os quais pode-se destacar: redução da fração de ejeção, aumento das câmeras, prolongamento do tempo de relaxamento isovolumétrico e redução da força de ejeção atrial. ${ }^{7}$ A miocardite pode estar acompanhada por outras manifestações cardíacas do LES, particularmente a pericardite e a endocardite; pode estar associada a miosite dos músculos esqueléticos. ${ }^{5} \mathrm{O}$ LES é comumente associado a doença arterial coronária (DAC), sendo essa a causa base da cardiomiopatia e devendo ser sempre excluída. Doenças crônicas, como hipertensão, podem inclusive acelerar ou contribuir para esse processo. ${ }^{6}$ A hidroxicloroquina está associada a uma forma rara e idiossincrática de cardiotoxicidade e a biópsia miocárdica pode ser necessária para descartar essa entidade. Os achados característicos da cardiotoxicidade por hidroxicloroquina podem ser vistos na microscopia óptica e na eletrônica. $\mathrm{Na}$ microscopia óptica pode ser encontrado a vacuolização difusa dos miócitos sem miocardite. Já na microscopia eletrônica encontra-se corpos sarcoplasmáticos mielinoides e curvilíneos. ${ }^{3}$

O tratamento da miocardite lúpica não foi avaliado em ensaios controlados. Foi observada melhora na função sistólica em alguns pacientes tratados com glicocorticoides, outros imunossupressores (ciclofosfamida, azatioprina) ou imunoglobulina intravenosa. Sugere-se que a miocardite lúpica aguda seja inicialmente tratada com altas doses de corticoides (metilprednisolona $1000 \mathrm{mg} /$ dia intravenosa por 3 dias, seguidos de $1 \mathrm{mg} / \mathrm{kg} / \mathrm{dia}$ ) associada a terapia usual para insuficiência cardíaca. ${ }^{3}$

O desfecho da miocardite lúpica é variável. Os pacientes com início agudo tendem a ter um desfecho mais favorável.

\section{REFERÊNCIAS}

1. Klumb EM, Silva CA, Lanna CC, Sato EI, Borba EF, Brenol JC, et al. Consenso da Sociedade Brasileira de Reumatologia para o diagnóstico, manejo e tratamento da nefrite lúpica. Rev Bras Reumatol. 2015;55(1):1-21.

2. Suri V, Varma S, Joshi K, Malhotra P, Kumari S, Jain S. Lupus myocarditis: marked improvement in cardiac function after intravenous immunoglobulin therapy. Rheumatol Int. 2010;30(11):1503-5.

3. Miner JJ, Kim AH. Cardiac manifestations of systemic lupus erythematosus. Rheum Dis Clin North Am. 2014;40(1):51-60.

4. Costenbader KH. Noncoronary cardiac manifestations of systemic lupus erythematosus in adults [Internet]. [S. 1.]: UoToDate; 2016
Comorbidades, como hipertensão arterial sistêmica, podem influenciar desfavoravelmente o prognóstico. Contudo, as conclusões sobre resultados a longo prazo de miocardite lúpica são baseadas principalmente em séries de casos individuais e, na maioria, a fração de ejeção melhora e a recorrência é rara. Os pacientes que se apresentam sintomáticos com insuficiência cardíaca congestiva, em sua maioria desenvolvem redução do débito ventricular, porém menos de $10 \%$ chegam a uma fração de ejeção $<30 \%{ }^{4}$

\section{CONCLUSÃO}

A miocardite é uma manifestação relativamente rara do LES, porém potencialmente fatal. A miocardite no LES é frequentemente assintomática, porém pode manifestar-se com febre, dispneia, palpitações e dor torácica ventilatório dependente. O diagnóstico depende principalmente da suspeita clínica e não de testes diagnósticos definitivos. ${ }^{4} \mathrm{Um}$ desfecho favorável depende de um reconhecimento rápido do quadro clínico e instituição rápida de um tratamento de suporte, imunossupressão e tratamento direcionado para a insuficiência cardíaca.

A paciente do caso apresentou, portanto, recorrência de um quadro incomum de miocardite lúpica isolada, em que ambas as vezes houve recuperação de uma fração de ejeção que chegou a ficar menor que $30 \%$. Tais quadros mostram-se na literatura com grandes índices de letalidade.

Assim, o envolvimento cardíaco por ter um amplo espectro de acometimento, necessita de uma cuidadosa investigação para o diagnóstico correto e precoce, a fim de que o paciente não atrase o tratamento e tenha maiores chances de reverter o quadro.

[acesso em: 20 jul 2016]. Disponível em: http://www.uptodate.com/ contents/non-coronary-cardiac-manifestations-of-systemic-lupuserythematosus-in-adults

5. Appenzeller S, Pineau CA, Clarke AE. Acute lupus myocarditis: clinical features and outcome. Lupus. 2011;20(9):981-8.

6. Zhang L, Zhu YL, Li MT, Gao N, You X, Wu QJ, et al. Lupus myocarditis: a case-control study from China. Chin Med J (Engl). 2015;128(19):2588-94.

7. Woo SI, Hwang GS, Kang SJ, Park JS, Park SJ, Lee YS, et al. Lupus myocarditis presenting as acute congestive heart failure: a case report. J Korean Med Sci. 2009;24(1):176-8.

\section{Como citar:}

Carneiro ML, Andrade TH, Ogawa MY, Braga FN. Miocardite lúpica, da insuficiência cardíaca severa à normalização da função cardíaca - relato de caso. Rev Med UFC. 2017 mai-ago;57(2):76-78. 